\title{
Ehrlichiosis: A Child With a Maculopapular Rash on His Trunk
}

\author{
Krishna Gollamudia, c, Roohab Shaikh ${ }^{\mathrm{b}}$
}

\begin{abstract}
Ehrlichiosis is a tick-borne infectious disease that is relatively uncommon; however, it has steadily increased in prevalence over the past 17 years. It is most frequently seen in Southeastern and Southcentral America with Oklahoma, Missouri, and Arkansas accounting for 30\% of the reported cases. Herein, we describe a 9-year-old male who was diagnosed with ehrlichiosis after a camping trip. He presented with a maculopapular rash, fatigue, fever and vomiting and the diagnosis was confirmed by a complete blood count which indicated leukopenia and thrombocytopenia. In conclusion, our case demonstrates the entire course of ehrlichiosis along with the various treatment options.
\end{abstract}

Keywords: Ehrlichiosis; Maculopapular rash; Trunk

\section{Introduction}

Ehrlichiosis is a tick-borne obligate intracellular bacterium that grows within membrane bound vacuoles in human leukocytes. The main reservoir is the white tail deer and whitefooted mouse, with the Lone Star tick (Amblyomma americanum) being the principal vector. Ehrlichiosis is characterized by headaches, myalgia, rash, nausea/vomiting, altered mental status, and lymphadenopathy. Minority of children have severe disease requiring them to be admitted to the ICU for ventilatory and hemodynamic support. Severe complications include seizures, coma, renal, respiratory and cardiac failure. Most ehrlichial diseases have an incubation period of 1 - 2 weeks, and symptoms appeared an average of 5.5 days after a tick bite was noted. It is diagnosed with an indirect fluorescent antibody test and commonly treated with a 10-day course of doxycycline.

Ehrlichiosis prevalence has steadily increased from 1994

Manuscript submitted May 30, 2017, accepted June 22, 2017

\footnotetext{
${ }^{a}$ Avalon University School of Medicine, 212 Churchill Hubbard Rd, Youngstown OH 44505, USA

bWindsor University School of Medicine, 6212 Monee Manhattan Rd, Monee, IL 60449, USA

${ }^{\mathrm{c} C o r r e s p o n d i n g ~ A u t h o r: ~ K r i s h n a ~ G o l l a m u d i, ~ A v a l o n ~ U n i v e r s i t y ~ S c h o o l ~ o f ~}$ Medicine, 212 Churchill Hubbard Rd, Youngstown OH 44505, USA.

Email: teja1989@gmail.com
}

doi: https://doi.org/10.14740/ijcp276w to 2010, when it became a reportable disease. Although it is easily treatable, ehrlichiosis tends to be commonly misdiagnosed for Rocky Mountain spotted fever or Lyme disease due to its common presentation. While these diseases have similar treatments, the lab work as well as the potential complications differs. Increasing ehrlichiosis awareness can lead to earlier diagnosis as well as decreased risk of complications.

\section{Case Report}

A 9-year-old male patient came to the clinic for a sick visit with his mother, exactly 1 week after Thanksgiving (December 1, 2016). For Thanksgiving break, the patient and his family went on a camping trip to Missouri. The mother first noticed a rash on his abdomen upon returning from their trip on Monday, 3 days prior to the visit, and stated she did not see any ticks. After returning home, the patient complained of a fever, appeared fatigued and the mother stated that he vomited twice. When questioned further, it was found that the family did not use any bug repellent on their trip. The mother claimed to have applied calamine lotion to the area with no relief, and gave the patient Tylenol and Motrin in an alternating pattern for temporary alleviation of symptoms. The patient had an albuterol inhaler, which he took as needed for his asthma but had no known allergies. The patient was born at 39 weeks gestation, through vaginal delivery, without complications. He met all his physical and developmental milestones, and was up to date with his immunizations. He attended the fourth grade and ac-

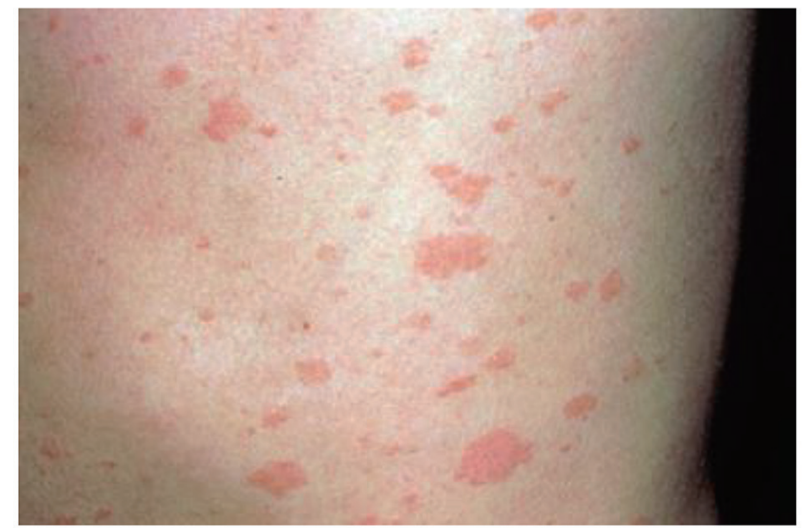

Figure 1. Erythematous maculopapular rash without distinct margins was seen. 
Table 1. Complete Blood Count Results

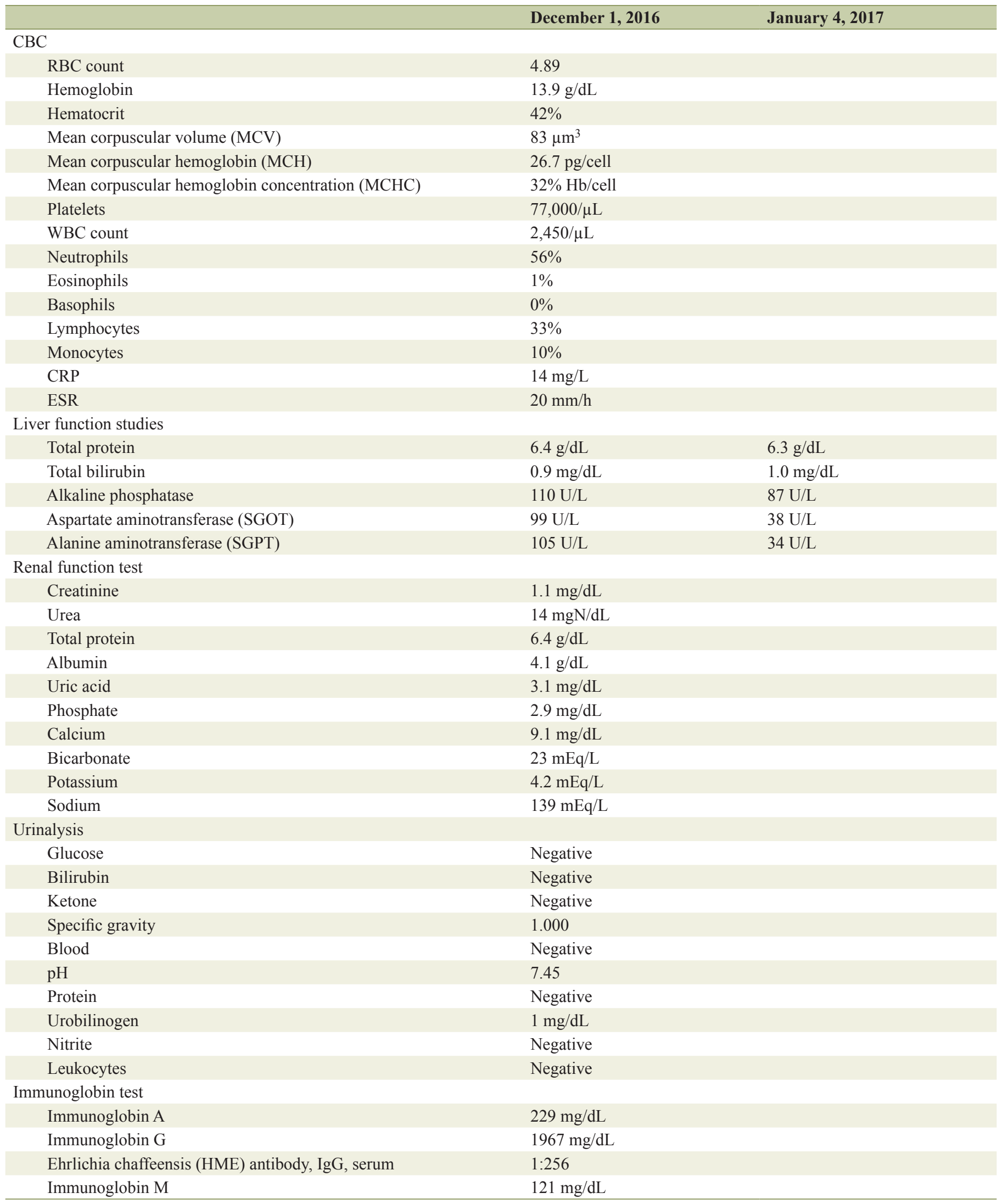


cording to his mother, was active and got along well with other children. The patient lives with his mother, father and younger sister in the suburbs of Chicago. His father has hypercholesterolemia, which is controlled by rosuvastatin and his mother suffers from hypothyroidism for which she takes levothyroxine. Upon physical examination, the patient was not in acute distress, his neck was supple and there was no lymphadenopathy. His temperature was $39.4^{\circ} \mathrm{C}\left(103^{\circ} \mathrm{F}\right)$, blood pressure was 106/64 mm Hg, and pulse was 96/min. Cardiovascular examination was unremarkable and there were no focal neurologic deficits. An extensive erythematous maculopapular rash without distinct margins was seen on his trunk (Fig. 1). A complete blood count (CBC) was performed which indicated leukopenia and thrombocytopenia (Table 1). The diagnosis was made based on the serology, which indicated elevated IgG antibodies for ehrlichiosis, as well as the clinical presentation and history. The patient was prescribed a 10-day course of doxycycline and mother was educated on the efficacy of DEET or tick repellents for their next camping trips. The child returned to the clinic exactly 34 days after the initial appointment (January 4), where his liver enzymes were back within a normal range. Upon further questioning, the mother stated that the rash had completely resolved by the ninth day of the antibiotic course.

\section{Discussion}

The boy is diagnosed with ehrlichiosis, a tick-borne obligate intracellular bacterium that grows within membrane bound vacuoles in human leukocytes. The main reservoir is the white tail deer and white-footed mouse, with the Lone Star tick ( $\mathrm{Am}$ blyomma americanum) being the principal vector [1]. Ehrlichiosis is characterized by headaches, myalgia, rash, nausea/ vomiting, altered mental status, and lymphadenopathy. Minority of children have severe disease requiring them to be admitted to the ICU for ventilatory and hemodynamic support. Severe complications include seizures, coma, renal, respiratory and cardiac failure. Most ehrlichial diseases have an incubation period of 1 - 2 weeks, and symptoms appeared an average of 5.5 days after a tick bite was noted [2].

Ehrlichiosis is commonly seen in males, people over 50 years of age, and immunocompromised patients [3]. However, many cases are also reported in children every year. The number of cases since have steadily increased from 200 cases in 2000, to 961 in 2008 [3]. The incidence rate was 3.2 cases per million person-years. The hospitalization rate was $57 \%$ and the case fatality rate was $1 \%$. The average reported annual incidence of HME in the United States was 3.2 cases per million population in 2012 , with wide variations in incidence by state. The mortality rate for ehrlichiosis is estimated to be around $2-5 \%$, with organ failure being the most likely cause of death [3]. Children are more prone to neurological deficits. Ehrlichiosis is most frequently reported in the Southeastern and Southcentral United States, from the Eastern seaboard extending westward to Texas. Three states (Oklahoma, Missouri, and Arkansas) account for $30 \%$ of all reported ehrlichiosis infections [3]. Occasionally, ehrlichiosis cases may be reported in other parts of the United States, including northern states where the Lone Star tick is not commonly found.

The differential diagnosis for ehrlichiosis is vast; however, it is most commonly confused with Rocky Mountain spotted fever. The differentiating factors are the lack of a rash and the presence of leukopenia. Lyme disease is also a common differential due to a similar rash presentation; however, leukopenia, thrombocytopenia and a high fever are not seen in Lyme disease [2]. Ehrlichiosis is less commonly mistaken for viral illnesses such as, mononucleosis, and Colorado tick fever.

The most common diagnostic procedure is the indirect fluorescent antibody test, which is estimated at $94-100 \%$ sensitive if the sample is obtained within 14 days following the onset of symptoms [2]. The detection of intracytoplasmic inclusions (morulae) in leukocytes is a quick method that may support the presumptive diagnosis [4]. The most common lab findings include leukopenia, elevated aminotransferases, lactate dehydrogenase, alkaline phosphatase and thrombocytopenia which are seen in majority of the patients [3]. Other findings may include anemia and an elevated plasma creatinine concentration.

\section{Consent}

Written informed consent was taken from the patient for publication of this case report and the accompanying images.

\section{References}

1. Statistics and Epidemiology - Ehrlichiosis. https://www. cdc.gov/ehrlichiosis/stats/. Published January 25, 2016. Accessed February 26, 2017.

2. Sexton DJ, McClain MT. Human ehrlichiosis and anaplasmosis. Human ehrlichiosis and anaplasmosis. https:// www.uptodate.com/contents/human-ehrlichiosis-andanaplasmosis? source $=$ search_result\&search; $=$ ehrlichiosi s\&selectedTitle;=1 48\#H16. Published August 15, 2016. Accessed February 26, 2017.

3. Symptoms, Diagnosis, and Treatment-Ehrlichiosis.https:// www.cdc.gov/ehrlichiosis/symptoms/. Published January 21, 2016. Accessed February 26, 2017.

4. Ganguly S, Mukhopadhayay SK. Tick-borne Ehrlichiosis infection in human beings. Vector Borne Dis 2008;45:273280. http://www.mrcindia.org/journal/issues/454273.pdf. 